\title{
Multimodal telepresent control of DLR's Rollin' JUSTIN
}

\author{
Philipp Kremer, Thomas Wimböck, Jordi Artigas, Simon Schätzle \\ Klaus Jöhl, Florian Schmidt, Carsten Preusche, Gerd Hirzinger
}

\begin{abstract}
This video presents a telepresence system which enables a human operator to explore a remote environment by means of a multimodal man machine interface and rollin' JUSTIN as teleoperator. The man machine interface allows for bimanual, dexterous manipulation and, through two different operating modi of the man machine interface, wide area movement as well. A bimanual assembly task, consisting of grasping a connector, opening and closing it again, is shown in this video.
\end{abstract}

\section{INTRODUCTION}

This video presents a multimodal telepresence system. Telepresence systems enable human operators to explore and act in remote environments by using a teleoperator. Reasons for using telepresence systems include scaling, environmental conditions, accessability, or spatial distance. Multimodal, bimanual, mobile telepresence systems, like the one proposed in [1], are developed for applications like minimale invasive surgery, telesurgery, training, rehabilitation, virtual assembly verification, virtual prototyping, micromanipulation, or satellite servicing. The remaining paper is structured in components, task and conclusion.

\section{COMPONENTS}

The man machine interface shown in figure 2 consists of two light weight robots [2], with grasping force controllers mounted as endeffectors for haptic feedback, and a head mounted display tracked by an optical tracking system for visual feedback. The teleoperator is DLR's rollin' JUSTIN. The components and their coupling are described in this section. All components communicate using a standard UDP/IP connection.

\section{A. Head Tracking and Visualization}

The human operator's head movements are tracked optically by a VICON system. This pose information is used to command JUSTIN's head pose. The stereo video cameras that are mounted inside JUSTIN's head are fed back to the human in order to provide visual feedback. The head mounted display is a Nvisor FX. The video projection in the background of figure 1 shows the view of one head camera of JUSTIN.

All authors are with the Institue of Robotics and Mechatronics, German Aerospace Center (DLR), 82230 Wessling, Germany philipp.kremeredlr.de

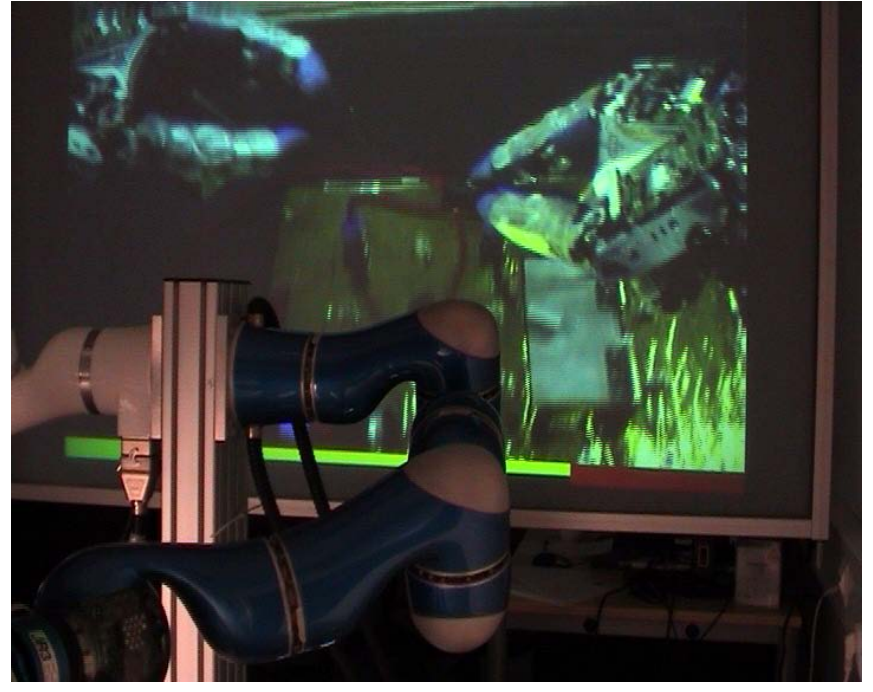

Fig. 1. The field of view of one eye of JUSTIN from the man machine interface

\section{B. Wide Area Movement}

Moving JUSTIN's base is enabled by switching to a second mode of the man machine interface. In this driving mode one robot of the man machine interface, usually the right arm, is used as a velocity command source. The endeffector of the robot can be moved like a scaled joystick so that translations and rotations in all directions are possible.

\section{Bilateral Arm Control}

The control scheme between master and slave arms is based on a four channel architecture. Both, 6 dimensional forces/torques and full space pose representations are sent in both directions. This allows better kinematic and dynamic haptic rendering performance, resulting in a more realistic and transparent behaviour compared to conventional position force architectures. By means of two virtual, spatial springs, one on each side, the desired cartesian forces are computed based on the error between present values of the local pose and that of the robot on the other side. Further, the master arms, configured with impedance causality, use force-torque sensors attached at the base of the grasping force controllers which permit the measurement of forces that are applied by the human hands. This force is not only locally fed to the master robot in order to reduce the effect of its own inertia, but is also used to drive the slave arm, which is equally configured with impedance causality. Using properly tuned gain factors for each control path allows for individual 
passivity of each, right and left arm system, and for reaching a desired degree of transparency.

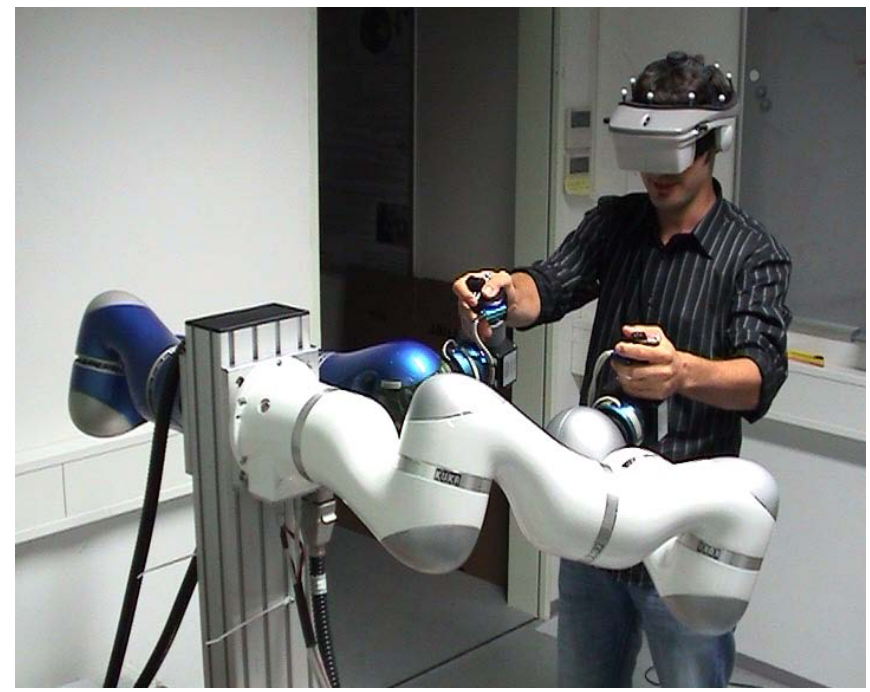

Fig. 2. A human operating the multimodal man machine interface

\section{Bilateral Hand Control}

To be able to command the robotic hands and feed back grasping forces, grasping force controllers are mounted at the light weight robots of the man machine interface. The one dimensional movement of the grasping force controller is mapped to different grasping trajectories, which are calculated dependent on the desired grip and starting positions of used robotic fingers. For the desired task, the right robotic hand uses thumb and index finger and the left one uses additionally the middle finger. The calculated Cartesian impedance forces from the robotic hand are fed back, the resulting one dimensional grasping force is calculated and then displayed to the human operator. The control architecture is described in [3].

\section{TASK}

The desired task involves some manipulations on a testboard positioned in front of JUSTIN, see figure 3. Only the fingertips are used for contacts as the task requires dexterity. The approaching phase, where the man machine interface operates in the driving mode, seems to be slow, because the human operator needs to look around in the remote environment. This is necessary, as automatic obstacle avoidance is not in use so far. The field of view is limited due to the used head mounted display and the cameras. Turning the head is consequently very important. The operator has to move in translational and rotational directions to reach the target, where different assembly tasks can be executed.

The task conducted is (A) grasping a connector which became loose in its clamp with a cable still securely attached to it. (B) Remove the cable from the connector by opening it and (C) reattach the cable. The connector is an umbilical type, which requires a translational movment at the beginning and afterwards, while still pulling it, a rotational movement.

To reinsert the connector visual marks on both sides are necessary. The stereo visualization provides enough depth vision to accomplish the reinsertion.

This task itself is quite complicated. Considering that the robotic hands are factor 1.5 bigger than averaged human hands and the connector is of unscaled size, the task is getting more challenging.

\section{SUMMARY}

This video presents a bimanual, dexterous telepresence system capable of wide area movements. The system's dexterity is demonstrated by opening and closing an umbilical type connector. Stereo-vision and force-feedback to the human operator increases the immersion into the remote environment and helps significantly that the operator can sucessfully fulfill such a two arm task.

\section{ACKNOWLEDGMENTS}

The authors gratefully acknowledge the contribution of National Research Organization and reviewers' comments.

\section{REFERENCES}

[1] A. Peer, Y. Komoguchi, and M. Buss, "Towards a mobile haptic interface for bimanual manipulations," in International Conference on Intelligent Robots and Systems, 2007, pp. 384-391.

[2] G. Hirzinger, N. Sporer, A. Albu-Schäffer, M. Hähnle, R. Krenn, A. Pascucci, and M. Schedl, "Dlr's torque-controlled light weight robot iii - are we reaching the technological limits now," in Proceedings of the IEEE Int. Conference Robotics Automation, 2002, pp. 1710-1716.

[3] A. Albu-Schäffer, O. Eiberger, M. Grebenstein, S. Haddadin, C. Ott, T. Wimböck, S. Wolf, and G. Hirzinger, "Soft robotics," IEEE Robotics and Automation Magazin, vol. 15, no. 3, pp. 20-30, 2008.

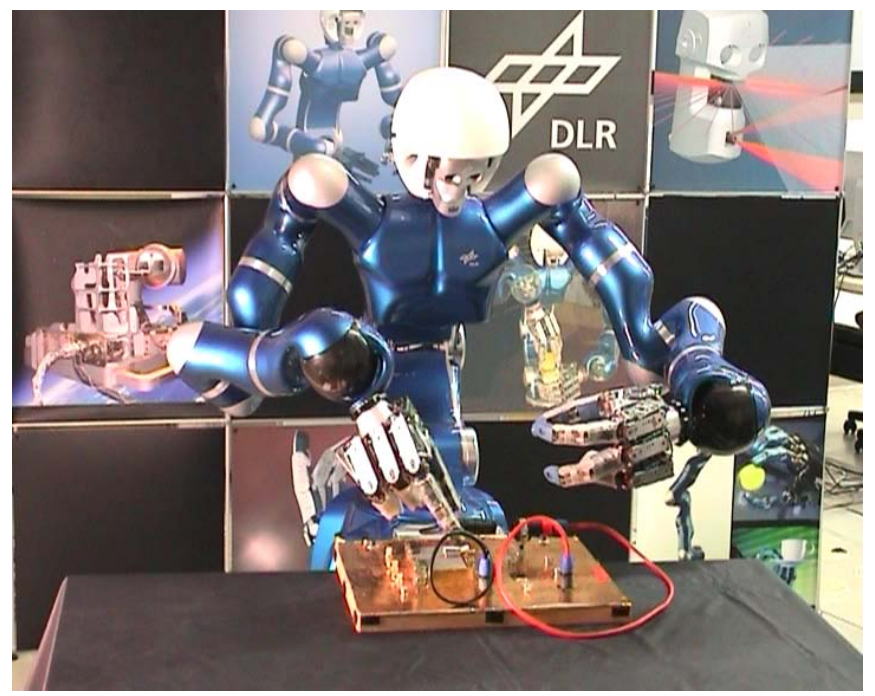

Fig. 3. JUSTIN handling a connector under telepresent control 